\title{
Exploring the entire conformational space of proteins by high-pressure NMR*
}

\author{
Kazuyuki Akasaka \\ Department of Biotechnological Science, School of Biology-Oriented Science and \\ Technology, Kinki University, Wakayama 649-6493, Japan and Cellular Signaling \\ Laboratory, RIKEN Harima Institute, Hyogo 679-5148, Japan
}

\begin{abstract}
A protein in solution is a thermodynamic entity, spanning, in principle, the entire allowed conformational space from the fully folded $\mathrm{N}$ to the fully unfolded $\mathrm{U}$. Although some alternately or partially folded higher-energy conformers may coexist with $\mathrm{N}$ and $\mathrm{U}$, they are seldom detected spectroscopically because their populations are usually quite low under physiological conditions. I describe here a new type of experiment, a combination of multidimensional NMR spectroscopy with pressure, that is capable of detecting and analyzing structures and thermodynamic stability of these higher-energy conformers. The idea is based on the finding that under physiological conditions the conformational order of a globular protein normally decreases in parallel with its partial molar volume (negative $\Delta V$ ), so that under equilibrium conditions, the population is shifted to a less-and-less-ordered conformer with increasing pressure. In principle, with the high space resolution of the multidimensional NMR, the method enables one to explore protein structure and stability in atomic detail in a wide conformational space from $\mathrm{N}$ to $\mathrm{U}$ with pressure and temperature as variables. The method will provide us with a strong basis for understanding the fundamental phenomena of proteins: function, folding, and aggregation.
\end{abstract}

\section{INTRODUCTION}

Unlike in crystals, protein in solution is a thermodynamic entity [1] existing, in principle, as a mixture of conformers differing in free energy within several $\mathrm{kcal} / \mathrm{mol}$. Understanding mechanisms of protein folding, function, as well as misfolding [2] requires the knowledge not only of the lowest-energy "native" conformer, but also of higher-energy "nonnative" conformers. However, higher-energy conformers are normally hidden behind the overwhelming population of the lowest-energy conformer except for some special cases [3], and, therefore, one cannot directly detect their presence by spectroscopic methods. One can detect their presence by utilizing a time-accumulation technique such as equilibrium hydrogen exchange [4] or else kinetically as transient species [5]. It is conceivable that they are distributed in a wide conformational space from the folded to the unfolded with a variety of conformation. Their direct spectroscopic detection, however, is only possible when their population is substantially increased to a level comparable to that of the basic native conformer. So far, no general method has been proposed for directly detecting and analyzing structures of nonnative conformers well outside the range of the basic native fold, except for those that are fully denatured.

Recently, pressure is gaining attention, as a perturbation that exerts quite different effects on protein conformation from that of temperature or solvent composition [6]. A protein in solution is, in gen-

\footnotetext{
*Plenary lecture presented at the $17^{\text {th }}$ IUPAC Conference on Chemical Thermodynamics (ICCT), Rostock, Germany, 28 July-2 August 2002. Other presentations are published in this issue, pp. 859-936.
} 
eral, a mixture of conformers differing not only in energy, but also in volume. Pressure acts on volume, namely, the partial molar volume of a protein. When pressure is applied to a protein solution, it can shift the conformational equilibrium in favor of a lower-volume conformer at the expense of a higher-volume conformer (Fig. 1, case 2) besides the general compression of each conformer (Fig. 1, case 1). As a result, the population of a lower-volume conformer may increase with increasing pressure. If this is combined with the atom-based resolution of two- or multidimensional NMR spectroscopy, one may be able to detect and analyze structures of less-ordered higher-energy conformers in a site-specific manner. This expectation has been realized by the introduction of the on-line cell high-pressure NMR technique [7] to the protein conformational study.

This paper starts with a description of the thermodynamic characteristics of the pressure-axis experiment on the conformational equilibrium of proteins, in particular, in contrast to the temperature-axis experiment. Then the volume theorem of protein is introduced, which leads to the generalization of the method to the conformational study of proteins in a wide conformational space. Finally, application of the method to several proteins will be reviewed, showing the ability of high-pressure NMR in clarifying the multiple conformational nature of globular proteins that are relevant to function, folding, and misfolding.

(1) General compression

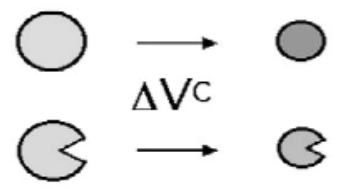

(2) Shift of Equilibrium

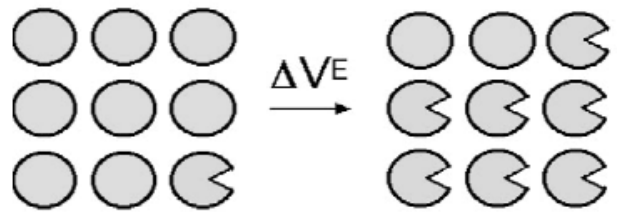

Fig. 1 Schematic presentation of the two ways that a protein can attain a lower volume in solution in a simple twostate equilibrium. 1. General compression within each conformational species $\left(\Delta V^{\mathrm{C}}\right)$. 2. Shift of equilibrium in favor of a lower-volume conformer $\left(\Delta V^{\mathrm{E}}\right)$.

\section{CHARACTERISTICS OF PRESSURE-AXIS EXPERIMENT: PRESSURE- VS. TEMPERATURE-AXIS EXPERIMENT}

Manipulating populations of nonnative conformers requires a shift of the conformational equilibrium (with an equilibrium constant $K$ ) by changing the free-energy difference between two conformers $\Delta G=-R T \ln K$. This may be attained by varying an external parameter, $P$ (pressure), $T$ (temperature), or $S$ (solvent composition, e.g., hydrogen ion or denaturant). The thermodynamic stability of a globular protein is normally expressed to the second order both in $T$ and $P$ by the equation,

$$
\begin{aligned}
& \Delta G(P)=\Delta G_{0}-\Delta S_{0}\left(T-T_{0}\right)-\Delta C_{p}\left(T-T_{0}\right)^{2} / 2 T_{0} \\
& +\Delta V_{0}\left(P-P_{0}\right)-\Delta \beta V\left(P-P_{0}\right)^{2} / 2+\Delta \alpha\left(P-P_{0}\right)\left(T-T_{0}\right)
\end{aligned}
$$


with changes in heat capacity $\Delta C_{p}$, compressibility coefficient $\Delta \beta$, expansivity $\Delta \alpha$, and $P_{0}$ normally taken at 1 bar, giving, in general, an ellipsoid in the $T-P$ plane [7]. The effect of $S$ parameter is included in $\Delta G_{0}$ itself. So far, perturbations in $T$ and $S$ axes have most frequently been used for this purpose.

Let us look into eq. 1 more carefully. First, in the temperature-axis experiment under constant pressure, the free-energy difference $\Delta G$ between two conformers results from a balance of two terms $\Delta H$ and $-T \Delta S$. Both $\Delta H$ and $\Delta S$ increase substantially with temperature. However, the first-order terms in $\Delta H$ and $-T \Delta S$ compensate each other. It is the second- (or higher-) order term of $\Delta H$ and $-T \Delta S$, rather than the first, that determines the $\Delta G$ profile on the temperature axis [8]. On the other hand, in the pressure-axis experiment (isothermal experiment) the free-energy difference $\Delta G$ between two conformers varies according to $P \Delta V_{0}-(\Delta \beta V / 2) P^{2}$, providing a basically nonlinear pressure dependence of $\Delta G$ (for a nonzero $\Delta \beta$ ). However, in practice, a clear nonlinear dependence of $\Delta G$ on pressure is seldom observed in the experimental plot of eq. 1 . This arises mainly from the fact that the contribution of the first-order term $P \Delta V_{0}$ is indeed large compared to that of the second-order term $(\Delta \beta V / 2) P^{2}$, because $\Delta V_{0}$ is large $(-20 \sim-200 \mathrm{ml} / \mathrm{mol})$ for most globular proteins particularly at physiological temperatures (below $\sim 37{ }^{\circ} \mathrm{C}$ ). Secondly, within the pressure range for which experimental data are normally available (500 bar $\sim 3 \mathrm{kbar}$ ), the number of data points are often limited to evaluate the second-order contribution. Although the evaluation of second-order and higher terms is certainly an important issue of protein physical chemistry, it is to be pursued by more rigorous experiments on relatively simple proteins. Thus, in practice, linear extrapolation is a reasonable approximation, making the extrapolation of $\Delta G$ to the physiological condition far simpler on the pressure axis than on the temperature axis, giving fairly reliable values of $\Delta G_{0}$ and $\Delta V_{0}$.

Another consequence of the temperature perturbation is that, because the protein system gains a substantial amount of internal energy $(\Delta H>100 \mathrm{kcal} / \mathrm{mol})$ [8] and entropy in thermal transition, any subtle conformational variations existing under physiological temperature are likely to be smeared out before they are detected spectroscopically. In contrast, the protein system gains only a total of several $\mathrm{kcal} / \mathrm{mol}$ at most through $P \Delta V$ within a normally employed pressure range of a few kbar. The additional, and yet practically quite important, merit of working on the pressure axis is that proteins behave practically reversibly under pressure and cause little problem from irreversible aggregation even after prolonged signal accumulation in two-dimensional NMR measurements. Such a situation is hard to be realized in the temperature-axis experiment.

\section{TWO WAYS TO CHOOSE LOWER VOLUME OF THE PROTEIN-WATER SYSTEM}

There are two ways for a protein solution to choose a lower volume under pressure; one by a general compression of the conformers present (defined with positive compressibility coefficient $\beta$ ) [9-11] and the other by a shift of equilibria among conformers in favor of lower-volume conformers $[12,13]$.

The two ways of volume change are shown schematically in Fig. 1. Here, a native conformer (represented by a circle, $\mathrm{N}$ ) and a lower-volume conformer (represented by a cracked circle, I) depict two conformers of the same protein. General compression by pressure (Fig. 1, case 1) occurs by shrinking the volume of each conformer $\left(V_{\mathrm{N}}\right.$ and $\left.V_{\mathrm{I}}\right)$ by $\Delta V^{\mathrm{C}}$. Shift of equilibrium by pressure (Fig. 1, case 2) occurs by increasing the equilibrium fraction of the cracked circle conformer by $\Delta V^{\mathrm{E}}$. In general, these two volume changes take place simultaneously, and the combined effects $\left(\Delta V^{\text {total }}=\Delta V^{\mathrm{C}}+\Delta V^{\mathrm{E}}\right)$ are detected as NMR spectral changes such as changes in chemical shift or intensity. In this article, we are interested in the shift of conformational equilibrium by pressure, because by using this effect one can increase the population of the normally invisible rare conformer (cracked circle; Fig. 1, case 2) dramatically and make it detectable and analyzable by NMR spectroscopy. Since the two events in Fig. 1 can occur simultaneously, separation of the two in the NMR spectrum is a crucial step for the structural analysis of the rare conformer. Furthermore, it is important to know how the structure of the rare conformer determined at high pressure is related to its structure at 1 bar. I will show below how these can be attained by noting that the general compression (Fig. 1, case 1) causes essentially a linear 
decrease in volume with pressure, whereas the shift of equilibrium (Fig. 1, case 2) causes a nonlinear decrease in volume with pressure.

\section{LINEAR COMPRESSION WITHIN THE SAME SUBENSEMBLE}

First, we look into the character of the general compression of a protein molecule, $\Delta V^{\mathrm{C}}$ (Fig. 1, case 1). Microscopically, compression of a protein volume is accompanied by slight changes in local conformations, which can be studied most sensitively by NMR as chemical shift changes. We found by highpressure NMR in solution that the general compression of the native conformer is accompanied by increased side chain packing $[14,15]$ and shortened hydrogen bond distances on the order of $\sim 0.02 \AA$ on average at $2 \mathrm{kbar}$ [16-21], accompanied by slight changes in torsion angles of a few degrees on average in $\phi, \psi$ angles [22], all of which occur heterogeneously over the folded protein architecture. The structural changes of comparable magnitude were reported in a crystalline protein at high pressure [10].

The important point here is that up to $2 \mathrm{kbar}$, the chemical shift changes of almost all ${ }^{1} \mathrm{H}$ and ${ }^{15} \mathrm{~N}$ nuclei in hen lysozyme and BPTI are linear with pressure [14-17,19-22]. This means that linear conformational changes take place with pressure in both secondary and tertiary structures, and hence a linear volume change in Fig. 1 within the measured pressure range $(<2 \mathrm{kbar})$, the efficiency of which is expressed by pressure-independent isothermal compressibility coefficient $\beta$ defined by

$$
\beta=-(1 / V)(\partial V / \partial P)
$$

where $V$ represents partial molar volume of a protein and $P$ represents pressure. The change in volume due to general compression is given by integrating eq. 2 from $P_{0}$ to $P$ by

$$
\Delta V^{\mathrm{C}}(P)=-\beta V\left(P-P_{0}\right)
$$

where $V$ is the partial molar volume of a protein, which is considered independent of pressure within the pressure range concerned. Compression means that $\beta$ is positive or that $\Delta V^{\mathrm{C}}$ is negative. Rigorously speaking, the compression should not be linear to the variation of pressure. The apparent linearity arises because the compression is quite small $(\sim 1 \%)$ [16] within a relatively small pressure range of $2-3 \mathrm{kbar}$. From the general relationship between compressibility and volume fluctuation [23], the pressure independence of compressibility means that the volume fluctuation is also independent of pressure. This would be expected only when the protein conformation at high pressure belongs essentially to the same conformational ensemble as at 1 bar. This leads to an important conclusion that the structure of a folded conformer at high pressure may be considered the same with the structure at $1 \mathrm{bar}$, except for a linear compression.

As to the effect of pressure on an unfolded conformer, we have less knowledge. However, we found that the ${ }^{1} \mathrm{H}$ and ${ }^{15} \mathrm{~N}$ chemical shifts of unfolded hen lysozyme in $8 \mathrm{M}$ urea [24] and a denatured melittin in water [25] are nearly completely linear with pressure up to 2 kbar. Furthermore, the effective hydrodynamic radius of the denatured state was not sensitive to pressure [24]. We take this as evidence showing that the volume of an unfolded conformer (the polypeptide chain plus water) is also decreased linearly with pressure, but the global conformation is not affected by pressure. Independently, a direct volumetric measurement with an oscillating densitometer showed that the partial molar volume of staphylococcal nuclease decreases linearly with pressure in the native folded as well as the heat-denatured unfolded state in the pressure range $1 \sim 700$ bar, with a nearly common compressibility coefficient $\beta$ for both cases [26]. These observations point to the notion that structural changes of the unfolded conformers occur also linearly with pressure, within the relatively small pressure range concerned.

Direct measurement of compressibility of intermediate conformers is difficult and rare, as they are not stable with a variation of pressure, or require a particular solvent condition for their production [27]. However, as both the native and fully unfolded conformers respond linearly to pressure, we may tentatively assume that intermediate conformers consisting of folded and unfolded parts should also re- 
spond linearly to pressure. In conclusion, we may generally expect in case 1 (Fig. 1) that, within a practical range of pressure $(1 \sim$ a few kbar), the structure of a conformer determined at high pressure is closely similar to the structure of that conformer at 1 bar.

\section{NONLINEAR COMPRESSION INVOLVING A TRANSITION BETWEEN DIFFERENT SUBENSEMBLES}

Next, we look into the character of the volume change of proteins under pressure due to a shift of equilibrium, $\Delta V^{\mathrm{E}}$ (Fig. 1, case 2). A protein in solution may generally consist of an equilibrium mixture of conformers with distinctly different structures or subensembles, e.g., the native, fully denatured, molten globule, locally unfolded intermediates [28], alternatively folded conformers [29,30] and low-lying excited state conformers [31-33], each having characteristic partial molar volume. Thus, the total volume of the protein system may also decrease by shifting the equilibrium in favor of lower-volume conformers (Fig. 1, case 2).

The volume change due to this effect is considered in more detail below for a simple case of an equilibrium between two conformers (subensembles), represented by the native conformer $\mathrm{N}$ and an intermediate conformer I, namely

$$
\mathrm{N} \Leftrightarrow \mathrm{I}
$$

The volume change $\Delta V^{\mathrm{E}}$ caused by the shift of the equilibrium can be expressed by the change in the equilibrium constant $K$, namely

$$
\Delta V^{\mathrm{E}}(P)=\Delta V_{0} /(1+1 / K)
$$

where $\Delta V_{0}=V_{\mathrm{I}}-V_{\mathrm{N}}$ is the difference in partial molar volume between conformers $\mathrm{N}$ and $\mathrm{I}$ at $P_{0}$ bar. $K$ is dependent on pressure $P$ according to

$$
K(P)=[\mathrm{I}] /[\mathrm{N}]=\exp (-\Delta G / R T)
$$

where $\Delta G=\Delta G_{\mathrm{I}}-\Delta G_{\mathrm{N}}$ is the Gibbs free-energy difference (the relative stability) between $\mathrm{N}$ and $\mathrm{I}$. Although, in general, the pressure dependence of $\Delta G$ is more complex [1], in a simple case in which the compressibility coefficients are nearly the same for N and I [26], $\Delta G$ is expressed as a linear function of $P$, namely

$$
\Delta G=\Delta G_{0}+\Delta V_{0}\left(P-P_{0}\right)
$$

$\Delta G_{0}$ is the difference in Gibbs energy at $P_{0}$ (meaning $\sim 1$ bar) and is normally positive ( $\mathrm{N}$ is more stable than I). The total partial molar volume change $\Delta V^{\text {total }}$ is given by the sum of the general compression $\Delta V^{\mathrm{C}}$ plus the volume change due to the shift of the equilibrium $\Delta V^{\mathrm{E}}$, namely

$$
\begin{aligned}
& \Delta V^{\text {total }}=\Delta V^{\mathrm{C}}+\Delta V^{\mathrm{E}}=-\beta V\left(P-P_{0}\right)+\Delta V_{0} /(1+1 / K) \\
& =-\beta V\left(P-P_{0}\right)+\Delta V_{0} /\left(1+\left\{\exp \left[\Delta G_{0} / R T+\Delta V_{0}\left(P-P_{0}\right) / R T\right]\right\}\right)
\end{aligned}
$$

which is depicted in Fig. 2. Since $\Delta V_{0}$ is usually negative (by a few tens to hundreds of $\mathrm{ml} / \mathrm{mol}$ ), the total volume decreases along the line as one increases the pressure. At sufficiently high pressure, the populations of $\mathrm{N}$ and I are reversed, and I becomes a dominant species instead of N. Pressure increases the population of a rare conformer I at the expense of the major conformer $N$ by virtue of the partial molar volume difference $\Delta V_{0}$. 


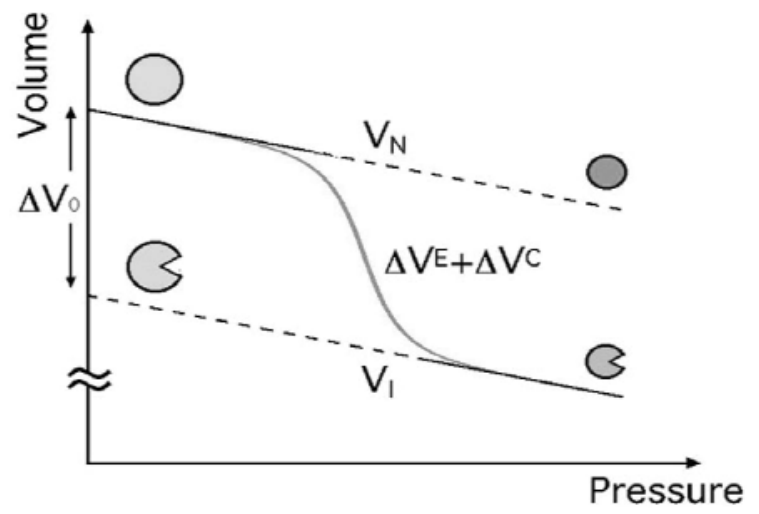

Fig. 2 Schematic representation showing how the total partial molar volume of a protein may change with pressure. Following a linear compression of $V_{\mathrm{N}}$ (the partial molar volume of the native conformer $\mathrm{N}$ ), a nonlinear (sigmoidal) volume change takes place as a result of the shift of equilibrium between $\mathrm{N}$ and $\mathrm{I}$, followed by a linear compression of $V_{\mathrm{I}}$ (the partial molar volumes of the intermediate conformer I).

\section{VOLUME THEOREM OF PROTEIN: PARTIAL MOLAR VOLUME PARALLELS CONFORMATIONAL ORDER}

The partial molar volume of a protein is, in general, contributed by the space occupied by constituent atoms, the cavities, and the change in volume of the solvent water due to hydration [34]. Here, we are interested only in the difference $\Delta V$ in partial molar volume rather than its absolute value. Although the magnitude of the volume contribution due to hydration is still obscure, recent experiments using sitespecific mutations show that the contribution from atom packing defects or cavities in the interior of the protein molecule is certainly one of the decisive factors determining $\Delta V_{0}$ [35,36]. Even a fair agreement is obtained between the experimentally determined $\Delta V_{0}$ by mutation and the $\Delta V_{0}$ predicted based on cavities in the $\mathrm{X}$-ray or NMR structures of the wild-type protein and the mutant, suggesting that cavities may indeed be a major factor in determining $\Delta V_{0}$. For more detailed discussions on factors determining volume changes in proteins, one may refer to a recent review [37].

Collapse of cavities is generally accompanied by their hydration. In fact, a globular protein can have a number of large and small cavities. The fully disordered protein, with all its cavities collapsed and hydrated, will have the lowest volume, and this is verified through eq. 6 , by the fact that most proteins undergo total unfolding at sufficiently high pressure. Intermediate conformers with local unfolding may have their larger cavities filled with water, but smaller cavities left intact, and therefore may have volumes in between the two. Thus, a distribution of conformers with varying partial molar volume may result under normal conditions of ambient temperature and pressure, depending on the degree of hydration of the cavities of the protein, namely, depending on the degree of conformational disorder. We may, therefore, put this into the volume theorem of protein: Partial molar volume of a protein decreases with the decrease of its conformational order. An experimental proof of the theorem comes from high-pressure NMR experiments carried out, so far, on several globular proteins under closely physiological conditions, which commonly show a distribution of conformers with decreasing partial molar volume in parallel with the loss of conformational order. However, the theorem is not rigorously proven, and, in fact, might not hold well in the high-temperature region where the volume contribution from the hydration layer is likely to become dominant [27]. 


\section{EXPLORING THE ENTIRE CONFORMATIONAL SPACE BY HIGH-PRESSURE NMR}

When a protein in solution exists in a mixture of various conformers differing in partial molar volume, a conformational transition like that in Fig. 2 may consecutively occur between any two conformers, as we increase the pressure, until the lowest-volume conformer is reached. This means that, in principle, a series of NMR spectra corresponding to a series of conformers may appear in the decreasing order of conformation, as the partial molar volume decreases with increasing pressure. Namely, we may scan the entire conformational space allowed for a protein from the fully ordered native to the totally disordered conformer under a given solution condition with pressure as sole variable. By combining this with twoor multidimensional NMR spectroscopy (e.g., ${ }^{15} \mathrm{~N} /{ }^{1} \mathrm{H} \mathrm{HSQC}$ ), a possibility is there that we may examine the structure of each intervening conformer with atom-based resolution. The experimental technique realizing this idea is the on-line cell high-pressure NMR technique with heteronuclear two- or multidimensional NMR capability recently introduced to protein studies $[1,38]$. The experiments performed so far with more than 10 proteins in a pressure range of $1 \sim 4000$ bar has shown that this idea is essentially realized [1,28-30,32,39-46]. This does not necessarily mean that all these intervening species give separately observable spectra as we increase the pressure, but some are separate and others appear mixed, depending on how rapidly they mutually exchange. Only when the exchange rate is much slower than the chemical shift separations of their signals, the two spectra are separately observed. However, when they exchange rapidly, the mixing would be sensed as nonlinear pressure dependence of chemical shift [32].

The nature of the high-pressure NMR experiment is better understood on the energy landscape of protein folding, namely the folding funnel $[47,48]$, which covers the entire conformational space allowed for a protein (Fig. 3). The depth of the funnel normally represents the conformational order (more rigorously, the internal energy of one protein molecule in water solvent) and the horizontal plane represents the conformational entropy of the polypeptide chain. The important point here is that, from the

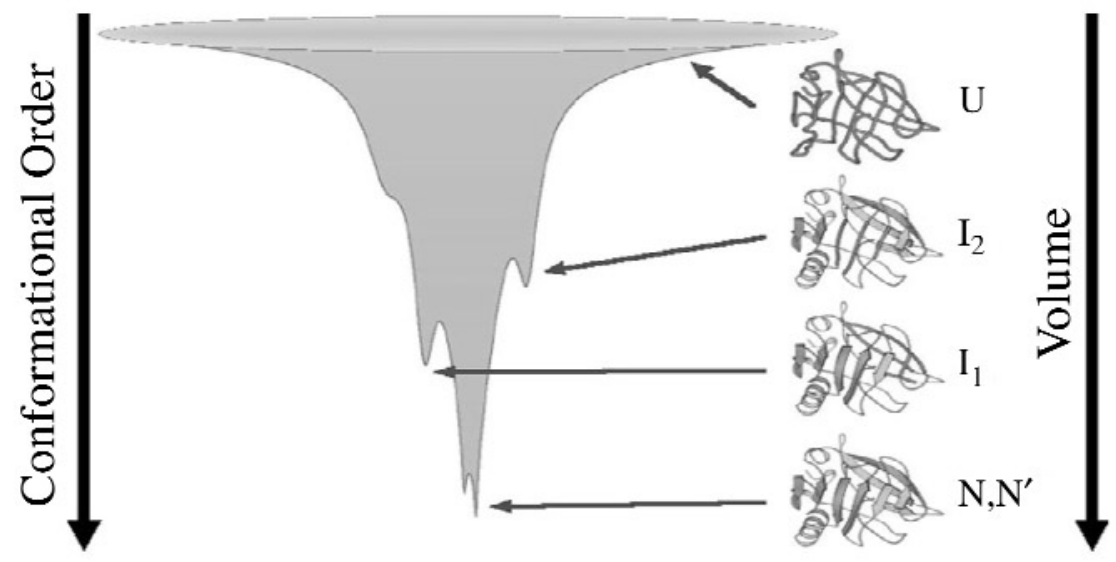

Fig. 3. The high-pressure NMR experiment depicted on a protein-folding funnel. The vertical axis shows the conformational order (increasing downward) and the horizontal plane shows conformational entropy. The partial molar volume of a protein is shown to change in parallel with the conformational order (the volume theorem, see text). The funnel is drawn schematically for $\beta$-lactoglobulin, with the five major conformational subensembles, the native $(N)$, the low-lying excited states $\left(N^{\prime}\right)$, intermediate states $\left(I_{1}, I_{2}\right)$ and the unfolded state $(U)$, identified in the high pressure NMR study [40], qualitatively placed in order of decreasing partial molar volume, $\mathrm{N}>\mathrm{N}^{\prime}>\mathrm{I}_{1}>\mathrm{I}_{2}>\mathrm{U}$. The structure of each subensemble is represented qualitatively on the native structure with the darkest lines representing disordered segments. These structures were deduced based on chemical shift and intensity analysis for ${ }^{1} \mathrm{H} /{ }^{15} \mathrm{~N}$ cross-peaks of individual amide groups on ${ }^{1} \mathrm{H} /{ }^{15} \mathrm{~N}$ HSQC two-dimensional NMR spectra under appropriate pressures. 
volume theorem considered valid under closely physiological conditions, a funnel can also be drawn with partial molar volume spanning the vertical axis, meaning that the variation in partial molar volume covers the entire conformational space of a protein (Fig. 3).

This particular funnel is drawn schematically for $\beta$-lactoglobulin [40], with the five major conformational sub ensembles, $\mathrm{N}, \mathrm{N}^{\prime}, \mathrm{I}_{1}, \mathrm{I}_{2}$, and $\mathrm{U}\left(\mathrm{N}\right.$; native, $\mathrm{N}^{\prime}$; alternatively folded conformer, $\mathrm{I}_{1}$; intermediate $1, \mathrm{I}_{2}$; intermediate 2, $\mathrm{U}$, fully unfolded), identified in the high-pressure NMR study [40]. All these conformers are considered in mutual equilibrium at any pressure from 1 to 2000 bar. From the mutual separation of cross peaks in the ${ }^{15} \mathrm{~N} /{ }^{1} \mathrm{H}$ HSQC spectra, we estimate that the exchange rate between $\mathrm{N}$ and $\mathrm{N}^{\prime}$ is rapid $\left(» 10^{3} \mathrm{~s}^{-1}\right)$ and the exchange rates involving the rest of the species are slow $\left(\ll 10^{3} \mathrm{~s}^{-1}\right)$. The structure of each conformer is qualitatively depicted on the native structure with the wide gray lines representing the structured part and the darkest lines symbolically representing disordered segments. These structures were obtained based on evaluation of individual cross-peak intensities as a function of pressure, giving simultaneously the information on volume changes $-57.4+/-14.4$ (from $\mathrm{N}$ to $\mathrm{I}_{1}$ ), $-90.0+/-35.2$ (from $\mathrm{N}_{\text {to }} \mathrm{I}_{2}$ ). The conformers are schematically arranged in the order of the experimentally determined order of partial molar volume, $V_{\mathrm{N}}>V_{\mathrm{N}^{\prime}}>V_{\mathrm{I} 1}>V_{\mathrm{I} 2}>V_{\mathrm{U}}$. We note, at least qualitatively, that the partial molar volume parallels the conformational order in $\beta$-lactoglobulin, consistently with the volume theorem.

\section{GENERALITY AND FUNCTIONAL RELEVANCE OF HIGHER-ENERGY CONFORMERS}

The example of $\beta$-lactoglobulin [40] above and other studies on the Ras-binding domain (RBD) of RalGDS [28], DHFR [29], ubiquitin [30], sperm whale apomyoglobin [41], P13 ${ }^{\text {MTCP1 }}$ [42], transthyretin [43], and hamster prion protein [44] showed that, by scanning pressure under suitable solution conditions in the range $1 \sim$ a few kbar, higher-energy conformers with varying degrees of structural order are produced. Often, the conformers range from the native fold to the complete unfolding, assisted in some cases by low temperature (such as $2 \sim-20{ }^{\circ} \mathrm{C}$ ) for enhanced unfolding. Structures of conformers appearing in the process were analyzed in site-specific detail based on pressure-induced shifts or intensity changes of basically all individual cross peaks in heteronuclear two-dimensional NMR spectra. The structures of nonnative conformers determined in this way are still fairly qualitative in the sense that no atomic coordinates are given. However, they are sufficient to discuss their roles in protein function as will be shown below.

The presence of open-barrel conforms $\left(I_{1}\right.$ and $\left.I_{2}\right)$ is considered important for $\beta$-lactoglobulin to bind various hydrophobic compounds in its barrel structure, and is likely to be a common design of lipocalin family proteins [40]. Similarly, a locally disordered conformer (I) and molten globule (MG) found in apomyoglobin seem also necessary for heme binding [41]. The local unfolding in RalGDSRBD is considered to be part of design of the signal transduction protein that mediates signal from one protein (or domain) to other protein (or domain) [28]. The alternative folded conformers found in DHFR and ubiquitin at high concentration (10 15\%) and in P13 ${ }^{\mathrm{MTCP} 1}$ are considered to be reactive forms of these proteins $[29,30,42]$. In contrast, so far we found no evidence of intermediate for amyloid-generating transthyretin, but only the fully unfolded monomers, which is likely to be a reactive form for amyloid formation [43]. Finally, hamster prion protein showed a locally disordered conformer to be present at about $1 \%$ under closely physiological condition, strongly suggesting that this be the "active" conformer turning into the contagious scrapie type. These examples show that a protein is indeed multiconformational and is designed by nature not just for the basic ground state, as is often considered, but for the higher-energy states that are functionally relevant.

\section{FUTURE DEVELOPMENT}

Higher-energy nonnative conformers of proteins have become targets of site-specific detail structural analysis by the introduction of the on-line cell high-pressure NMR technique. So far, the structure has 
been determined based on pressure-induced shifts or intensity changes of basically all individual crosspeaks in heteronuclear two-dimensional NMR spectra. However, the structures determined in this way are still fairly qualitative in the sense that no atomic coordinates are given. The higher-energy structures are themselves subensembles of generally more flexible conformers than their lowest-energy counterpart, and expressing their structures in fixed coordinates may often be unappropriate. However, in some cases, it would be better to determine average coordinates of structures following the established routine. Technically, in near future, a significant increase in signal-to-noise ratio is anticipated by increasing the sample volume and the use of a cryo-probe, which will enable us to incorporate nuclear Overhauser effect constraints to the elucidation of average atomic coordinates of nonnative conformers. In parallel with this, a new approach using pressure-induced chemical shifts for structural determination has emerged [49]. In conclusion, we have now a promising general means for exploring the entire conformational space of a protein and determining nonnative structures in atomic details along with their thermodynamic stability.

\section{REFERENCES}

1. C. B. Anfinsen. Science 181, 223-230 (1973).

2. C. M. Dobson. Trends Biochem. Sci. 24, 329-332 (1999).

3. P. E. Wright and H. J. Dyson. J. Mol. Biol. 293, 321-331 (1999).

4. C. Woodward, I. Simon, E. Techsen. Mol. Cell. Biochem. 48, 135-160 (1982).

5. S. W. Englander and L. Mayne. Ann. Rev. Biophys. Biomol. Struct. 21, 243-265 (1992).

6. J. L. Silva, D. Foguel, C. A. Royer. Trends Biochem. Sci. 26, 612-618 (2001).

7. K. Akasaka and H. Yamada. Methods in Enzymology 338: Nuclear Magnetic Resonance of Biological Macromolecules Part A, T. L. James, V. Doetsch, U. Schmitz (Eds.), pp. 134-158, Academic, New York (2001).

8. P. L. Privalov and S. J. Gill. Adv. Protein Chem. 39, 191-234 (1988).

9. K. Gekko and Y. Hasegawa. Biochemistry 25, 6563-6571 (1986).

10. C. E. Kundrot and F. M. Richards. J. Mol. Biol. 193, 157-170 (1987).

11. D. P. Kharakoz. Biochemistry 36, 10276-10285 (1997).

12. S. A. Hawley. Biochemistry 10, 2436-2442 (1971).

13. J. Jonas, L. Ballard, D. Nash. Biophys. J. 75, 445-452 (1998).

14. K. Akasaka, T. Tezuka, H. Yamada. J. Mol. Biol. 271, 671-678 (1997).

15 H. Li, H. Yamada, K. Akasaka. Biophys. J. 77, 2801-2812 (1999).

16. H. Li, H. Yamada, K. Akasaka. Biochemistry 37, 1167-1173 (1998).

17. K. Inoue, H. Yamada, T. Imoto, K. Akasaka. J. Biomol. NMR 12, 535-541 (1998).

18. H.-R. Kalbitzer, A. Gorler, H. Li, P. V. Dubovskii, W. Hengstenberg, C. Kowolik, H. Yamada, K. Akasaka. Protein Sci. 9, 693-703 (2000).

19. M. Iwadate, T. Asakura, P. V. Dubovskii, H. Yamada, K. Akasaka, M. P. Williamson. J. Biomol. NMR 19, 115-124 (2001).

20. H. Li, H. Yamada, K. Akasaka, A. M. Gronenborn. J. Biomol. NMR 18, 207-216 (2000).

21. M. Marchi and K. Akasaka. J. Phys. Chem. B 105, 711-714 (2001).

22. K. Akasaka, H. Li, H. Yamada, R. Li, T. Thoresen, C. K. Woodward. Protein Sci. 8, 1946-1953 (1999).

23. A. Cooper. Proc. Natl. Acad. Sci. USA 73, 2740-2741 (1976).

24. Y. O. Kamatari, H. Yamada, K. Akasaka, J. A. Jones, C. M. Dobson, L. J. Smith. Eur. J. Biochem. 268, 1782-1793 (2001).

25. K. Akasaka, H. Li, P. Dubovskii, H. R. Kalbitzer, H. Yamada. "High resolution-high pressure NMR spectroscopy. Application to protein structure, dynamics and folding", in Structure, Dynamics and Function of Biological Macromolecules, O. Jardetzky and M. D. Finucane (Eds.), pp. 77-92, IOS Press, Amsterdam (2001). 
26. H. Seemann, R. Winter, C. A. Royer. J. Mol. Biol. 307, 1091-1102 (2001).

27. T. V. Chalikian and K. J. Breslauer. Biopolymers 39, 619-625 (1996).

28. K. Inoue, H. Yamada, K. Akasaka, C. Herrmann, W. Kremer, T. Maurer, R. Döker, H. R. Kalbitzer. Nat. Struct. Biol. 7, 547-550 (2000).

29. R. Kitahara, S. Sareth, H. Yamada, E. Ohmae, K. Gekko, K. Akasaka. Biochemistry 39, 12789-12795 (2000).

30. R. Kitahara, H. Yamada, K. Akasaka. Biochemistry 40, 13555-13563 (2001).

31. N. J. Baxter, L. L. P. Hosszu, J. P. Waltho, M. P. Williamson. J. Mol. Biol. 284, 1625-1639 (1998).

32. K. Akasaka and H. Li. Biochemistry 40, 8665-8671(2001).

33. F. A. A. Mulder, A. Mittermaier, B. Hon, F. W. Dahlquist, L. E. Kay. Nature Struct. Biol. 8, 932-935 (2001).

34. K. Gekko. "Partial specific (molar) volume", in The Encyclopedia of Molecular Biology, T. E. Creighton (Ed.), Wiley, New York (1999).

35. K. J. Frye and C. A. Royer. Protein Sci. 7, 2217-2222 (1998).

36. M. W. Lassalle, H. Yamada, K. Ogata, A. Sarai, K. Akasaka. Proteins: Struct., Funct. Genet. 45, 96-101 (2001).

37. C. A. Royer. Biochim. Biophys. Acta 1595, 201-209 (2002).

38. J. L. Urbauer, M. R. Ehrhardt, R. J. Bieber, P. F. Flynn, A. J. Wand. J. Am. Chem. Soc. 118, 11329-11330 (1996).

39. K. Inoue, T. Maurer, H. Yamada, C. Herrmann, G. Horn, H. R. Kalbitzer, K. Akasaka. FEBS Lett. 506, 180-184 (2001).

40. K. Kuwata, H. Li, H. Yamada, C. A. Batt, Y. Goto, K. Akasaka. J. Mol. Biol. 305, 1073-1083 (2001).

41. R. Kitahara, H. Yamada, K. Akasaka, P. E. Wright. J. Mol. Biol. 320, 311-319 (2002).

42. R. Kitahara, C. Royer, H. Yamada, M. Boyer, J. L. Saldana, K. Akasaka, C. Roumestand. J. Mol. Biol. 329, 609-628 (2002).

43. T. N. Niraula, K. Haraoka, Y. Ando, H. Li, H. Yamada, K. Akasaka. J. Mol. Biol. 320, 333-342 (2002).

44. K. Kuwata, H. Li, H. Yamada, G. Legname, S. B. Prusiner, K. Akasaka, T. L. James. Biochemistry 41, 12277-12283 (2002).

45. M. W. Lassalle, H. Li, H. Yamada, K. Akasaka. Protein Sci. 12, 66-72 (2003).

46. R. Kitahara and K. Akasaka. Proc. Natl. Acad. Sci. USA 100, 3167-3172 (2003).

47. K. A. Dill and H. S. Chan. Nat. Struct. Biol. 4, 10-19 (1997).

48. K. A. Dill. Protein Sci. 8, 1166-1180 (1999).

49. R. Refaee, T. Tezuka, K. Akasaka, M. P. Williamson. J. Mol. Biol. 327, 857-865 (2003). 